\section{Emergency Radiology COFFEE Case Book: Case-Oriented Fast Focused Effective Education}

B. Khurana, J. Mandell, A. Sarma, S. Ledbetter, eds.

Cambridge, U.K.: Cambridge University Press, 2016, 668 pages, $\$ 155$

Emergency radiology is a subspecialty of radiology, as well as a rapidly growing area of clinical practice, that specializes primarily in the use of diagnostic imaging in the acutely ill or traumatized patient. It aligns the clinical practice of radiology with the referring services of emergency medicine and surgery and requires proper application of a diverse body of knowledge across multiple radiologic subspecialties. The goal of emergency radiology is to rapidly provide a high-quality, final imaging interpretation in all emergent cases, including both inpatients and patients in the emergency department.

The aim of this book is to quickly augment the competency of its readers in this core discipline. The 4 editors and 15 contributors coined the acronym COFFEE (Case-Oriented Fast Focused Effective Education) to describe an image-rich, case-based diagnostic approach emphasizing the spectrum of a disease and differential diagnosis - an approach that, in the early 2000s, was found to be a fast and effective educational method. The 85 index cases are organized into two parts (nontraumatic, with abdominal, thoracic, otolaryngologic, neurologic, and musculoskeletal sections, and traumatic, with neurologic, thoracic, abdominal, and musculoskeletal sections) and are portrayed in a way that simulates real-life radiology practice in the emergency department. Each chapter begins with an index case, which is presented as an unknown in a standardized format that includes a summary of the major teach- ing points, a description of disease variants and clinical or imaging management, instructional figures or illustrations, and the differentiation. A self-assessment provides additional instruction about the significance of specific clinical and imaging findings. Companion cases spanning the differential diagnoses and spectrum of the disease provide hundreds of additional examples with highquality images. Many lesions or anatomic landmarks on the images are clearly indicated by colorful arrows. There are updated references at the end of each chapter for further reading, as well as a helpful index.

This book is intended to be a go-to resource for both radiologists and clinicians, to improve their performance and confidence in interpreting images during their active work. It will also be helpful for nuclear medicine physicians and trainees in interpreting PET/CT, SPECT/CT, or PET/MRI studies. It should be highly useful for every emergency department physician and should be a part of every emergency medicine resident's personal library. It is also an excellent resource for radiology or emergency medicine residents studying for board certification.

\section{E. Edmund Kim \\ University of California at Irvine 101 The City Dr. S. Orange, CA 92868 E-mail: edmundek@uci.edu}

Published online Oct. 6, 2016

DOI: 10.2967/jnumed.116.184770

COPYRIGHT (c) 2016 by the Society of Nuclear Medicine and Molecular Imaging, Inc. 